\begin{tabular}{ll} 
C O L L O Q U I U M & M A T H E M A T I C U M \\
\hline VOL. LXV & $\frac{1993}{\text { FASC. } 2}$ \\
\hline
\end{tabular}

\title{
LOWER BOUNDS FOR THE SOLUTIONS IN THE SECOND CASE OF FERMAT'S EQUATION WITH PRIME POWER EXPONENTS
}

BY

MAOHUA LE (CHANGSHA)

Let $p$ be an odd prime, and let $n$ be a positive integer. Further, let $x, y$, $z$ be integers satisfying

$$
x^{p^{n}}+y^{p^{n}}=z^{p^{n}}, \quad p \mid x y z, \quad 0<x<y<z, \quad \operatorname{gcd}(x, y)=1 .
$$

Recently, Zhong [2] proved that $y>p^{3 n p^{n}-n} / 2$ and $z-x>p^{3 n p^{n}-n-1} / 4$. In this note we partly improve the above result as follows:

THEOREM. If $p \equiv 3(\bmod 4)$, then $y>p^{6 n p^{n}-3 n^{2}-2 n+3} / 2^{1 / p^{n}}$ and $z-$ $x>p^{6 n p^{n}-3 n^{2}-3 n+3} / 2^{1-1 / p^{n}}$.

Proof. It is a well known fact that (1) is impossible for $p=3$, so we may assume that $p>3$.

We first deal with the case that $p \mid x$. Let $p^{\alpha} \| x$. Then from (1) we get

$$
\begin{gathered}
z-y=p^{\alpha p^{n}-n} x_{0}^{p^{n}}, \\
\frac{z^{p^{i}}-y^{p^{i}}}{z^{p^{i-1}}-y^{p^{i-1}}}=p x_{i}^{p^{n}}, \quad i=1, \ldots, n,
\end{gathered}
$$

where $x_{0}, x_{1}, \ldots, x_{n}$ are positive integers satisfying $p \nmid x_{0} x_{1} \ldots x_{n}$ and

$$
x=p^{\alpha} x_{0} x_{1} \ldots x_{n} .
$$

For any coprime integers $X, Y$, by the proof of the Theorem in [1], we find that if $p \equiv 3(\bmod 4)$ then $\left(X^{p}-Y^{p}\right) /(X-Y)=A^{2}+p B^{2}$, where $A$, $B$ are integers satisfying $\operatorname{gcd}(A, B)=1$ and $A \equiv 0(\bmod (X-Y))$. Hence, by (3), we have

$$
\frac{z^{p^{i}}-y^{p^{i}}}{z^{p^{i-1}}-y^{p^{i-1}}}=A_{i}^{2}+p B_{i}^{2}=p x_{i}^{p^{n}}, \quad i=1, \ldots, n
$$

1991 Mathematics Subject Classification: Primary 11D41.

Supported by the National Natural Science Foundation of China. 
whence we get

$$
B_{i}^{2}+p\left(\frac{A_{i}}{p}\right)^{2}=x_{i}^{p^{n}}, \quad i=1, \ldots, n,
$$

where $A_{i}, B_{i}(i=1, \ldots, n)$ are integers satisfying $\operatorname{gcd}\left(A_{i}, B_{i}\right)=1$ and

$$
A_{i} \equiv 0\left(\bmod \left(z^{p^{i-1}}-y^{p^{i-1}}\right)\right), \quad i=1, \ldots, n .
$$

Further, by $(2), A_{i} / p(i=1, \ldots, n)$ are integers satisfying

$$
\frac{A_{i}}{p} \equiv 0\left(\bmod p^{\alpha p^{n}-n+i-2}\right), \quad i=1, \ldots, n .
$$

Notice that $p>3$ and the class number of the imaginary quadratic field $\mathbb{Q}(\sqrt{-p})$ is less than $p$. By an argument similar to the proof of the Theorem in [1], we see from (5) that there exist integers $X_{i}, Y_{i}(i=1, \ldots, n)$ satisfying

$$
x_{i}=X_{i}^{2}+p Y_{i}^{2}, \quad \operatorname{gcd}\left(X_{i}, Y_{i}\right)=1, \quad i=1, \ldots, n,
$$

and

$$
B_{i}+\frac{A_{i}}{p} \sqrt{-p}=\left(X_{i}+Y_{i} \sqrt{-p}\right)^{p^{n}}, \quad i=1, \ldots, n .
$$

From (8),

(9) $\frac{A_{i}}{p}=p^{n} Y_{i} \sum_{j=0}^{\left(p^{n}-1\right) / 2}(-1)^{j}\left(\begin{array}{c}p^{n} \\ 2 j+1\end{array}\right) p^{j-n} X_{i}^{p^{n}-2 j-1} Y_{i}^{2 j}, \quad i=1, \ldots, n$.

Notice that if $p>3$ and $j>0$, then $j>(\log (2 j+1)) / \log p$ and

$$
\left(\begin{array}{c}
p^{n} \\
2 j+1
\end{array}\right) p^{j-n}=\left(\begin{array}{c}
p^{n}-1 \\
2 j
\end{array}\right) \frac{p^{j}}{2 j+1} \equiv 0(\bmod p) .
$$

Since $p \nmid x_{i}(i=1, \ldots, n)$, we have $p \nmid X_{i}(i=1, \ldots, n)$ by $(7)$, and hence

$$
Y_{i} \equiv 0\left(\bmod p^{\alpha p^{n}-2 n+i-2}\right), \quad i=1, \ldots, n,
$$

by (6) and (9). Since $x_{i}>1(i=1, \ldots, n)$, we have $Y_{i} \neq 0(i=1, \ldots, n)$ by (7). Thus, we obtain

$$
x_{i}>p^{2 \alpha p^{n}-4 n+2 i-3}, \quad i=1, \ldots, n,
$$

by (7) and (10), and hence

$$
x>p^{\alpha+\Sigma_{i=1}^{n}\left(2 \alpha p^{n}-4 n+2 i-3\right)}=p^{2 \alpha n p^{n}-3 n^{2}-2 n+\alpha}
$$

by (4). Notice that $\alpha \geq 3$ by [2]. We get $x>p^{6 n p^{n}-3 n^{2}-2 n+3}$ by (11).

Using the same method, we can prove that $y>p^{6 n p^{n}-3 n^{2}-2 n+3}$ and $z>p^{6 n p^{n}-3 n^{2}-2 n+3}$ correspond to $p \mid y$ and $p \mid z$ respectively. Thus, $y>$ 
$p^{6 n p^{n}-3 n^{2}-2 n+3} / 2^{1 / p^{n}}$ since $2^{1 / p^{n}} y>z$. Simultaneously, we have

$$
\begin{aligned}
z-x & =\frac{y^{p^{n}}}{z^{p^{n}-1}+x z^{p^{n}-2}+\ldots+x^{p^{n}-1}}>\frac{y^{p^{n}}}{p^{n} z^{p^{n}-1}} \\
& >\frac{y^{p^{n}}}{p^{n}\left(2^{1 / p^{n}} y\right)^{p^{n}-1}}=\frac{y}{2^{1-1 / p^{n}} p^{n}}>p^{6 n p^{n}-3 n^{2}-3 n+3} / 2^{1-1 / p^{n}} .
\end{aligned}
$$

The theorem is proved.

\section{REFERENCES}

[1] M.-H. Le, Lower bounds for the solutions in the second case of Fermat's last theorem, Proc. Amer. Math. Soc. 111 (1991), 921-923.

[2] C.-X. Zhong, On Fermat's equation with prime power exponents, Acta Arith. 59 (1991), 83-86.

Current address:

RESEARCH DEPARTMENT

CHANGSHA RAILWAY INSTITUTE

CHANGSHA, HUNAN

P.R. CHINA
DEPARTMENT OF MATHEMATICS HUNAN NORMAL UNIVERSITY

P.O. BOX 410081 CHANGSHA, HUNAN 